C. R. Acad. Sci. Paris, t. 2, Série IV, p. 1-??, 2001

- PXHY1171.TEX -

Rubrique/Heading

(Sous-rubrique/Sub-Heading)

\title{
On the two-body problem in general relativity
}

\author{
Luc Blanchet ${ }^{\text {a }}$ \\ a Institut d'Astrophysique de Paris, 98bis boulevard Arago, 75014 Paris, France \\ E-mail: blanchet@iap.fr
}

(Reçu le jour mois année, accepté après révision le jour mois année)

\begin{abstract}
We consider the two-body problem in post-Newtonian approximations of general relativity. We report the recent results concerning the equations of motion, and the associated Lagrangian formulation, of compact binary systems, at the third post-Newtonian order $\left(\sim 1 / c^{6}\right.$ beyond the Newtonian acceleration). These equations are necessary when constructing the theoretical templates for searching and analyzing the gravitational-wave signals from inspiralling compact binaries in VIRGO-type experiments. (C) 2001 Académie des sciences/Éditions scientifiques
\end{abstract} et médicales Elsevier SAS

general relativity/equations of motion

Sur le problème des deux corps en relativité générale

Résumé. $\quad$ Nous considérons le problème des deux corps dans l'approximation post-newtonienne de la relativité générale. Nous présentons les résultats récents concernant les équations du mouvement, et la formulation lagrangienne associée, de systèmes binaires d'objets compacts, au troisième ordre post-newtonien $\left(\sim 1 / c^{6}\right.$ après l'accélération newtonienne). Ces équations sont utilisées dans la construction des filtres théoriques pour la détection et l'analyse des signaux d'ondes gravitationnelles provenant des binaires compactes spiralantes dans les expériences du type VIRGO. (C) 2001 Académie des sciences/Éditions scientifiques et médicales Elsevier SAS

relativité générale/équations du mouvement

$\overline{\text { Note présentée par First name NAME }}$

S1296-2147(01)01171-?/FLA

(C) 2001 Académie des sciences/Éditions scientifiques et médicales Elsevier SAS. Tous droits réservés. 1 


\section{Luc Blanchet}

By two-body problem we mean the problem of the dynamics of two strutureless, non-spinning point-particles, characterized by solely two mass parameters $m_{1}$ and $m_{2}$, moving under their mutual, purely gravitational, interaction. Surely this problem, because of its conceptual simplicity, is among the most interesting ones to be solved within any theory of gravity. Actually, there are two aspects of the problem: the first sub-problem consists of obtaining the equation of the binary motion, the second is to find the (hopefully exact) solution of that equation. We refer to the equation of motion as the explicit expression of the acceleration of each of the particles in terms of their positions and velocities. It is well known that in Newtonian gravity, the first of these sub-problems is trivial, as one can easily write down the equation of motion for a system of $N$ particles, while the second one is difficult, except in the two-body case $N=2$, which represents in fact the only situation amenable to an exact treatment of the solution.

In general relativity, even writing down the equations of motion in the simplest case $N=2$ is difficult. Unlike in Newton's theory, it is impossible to express the acceleration by means of the positions and velocities, in a way which would be valid within the "exact" theory. Therefore we are obliged to resort to approximation methods. Let us feel reassured that plaguing the exact theory of general relativity with approximation methods is not a shame. It is fair to say that many of the great successes of this theory, when confronted to experiments and observations, have been obtained thanks to approximation methods. Furthermore, the beautiful internal wheels of general relativity also show up when using approximation methods, which often deserve some theoretical interest in their own, as they require interesting mathematical techniques.

Here we whall investigate the equation of the binary motion in the post-Newtonian approximation, i.e. as a formal expansion when the velocity of light $c$ tends to infinity, up to the so-called third post-Newtonian (3PN) order, i.e. $1 / c^{6}$ beyond the usual Newtonian acceleration. This problem is not pure academicism, because the equation of motion at this somewhat frightening $3 \mathrm{PN}$ order is needed (among other things) during the computation of the orbital phase of the inspiralling compact binaries [1 2 . 2. The phase constitutes the crucial component of the theoretical templates to be used for searching and analyzing the gravitational waves from the binary inspiral in VIRGO-type detectors.

We write the $3 \mathrm{PN}$ acceleration of the particle 1 , say, in the form

$$
a_{1}^{i}=A_{\mathrm{N}}^{i}+\frac{1}{c^{2}} A_{1 \mathrm{PN}}^{i}+\frac{1}{c^{4}} A_{2 \mathrm{PN}}^{i}+\frac{1}{c^{5}} A_{2.5 \mathrm{PN}}^{i}+\frac{1}{c^{6}} A_{3 \mathrm{PN}}^{i}+\mathcal{O}\left(\frac{1}{c^{7}}\right)
$$

where the first term is given by the famous Newtonian law,

$$
A_{\mathrm{N}}^{i}=-\frac{G m_{2}}{r_{12}^{2}} n_{12}^{i}
$$

For simplicity, we do not write the particle's label 1 on the $A_{\mathrm{nPN}}^{i}$ 's. We denote by $r_{12}=\mid \mathbf{y}_{1}(t)-$ $\mathbf{y}_{2}(t) \mid$ the distance between the two particles, with $\mathbf{y}_{1}=\left(y_{1}^{i}\right)$ and $\mathbf{y}_{2}=\left(y_{2}^{i}\right)$ their "absolute" positions, by $n_{12}^{i}=\left(y_{1}^{i}-y_{2}^{i}\right) / r_{12}$ the corresponding unit direction, and by $a_{1}^{i}=d v_{1}^{i} / d t$ and $v_{1}^{i}=d y_{1}^{i} / d t$ the acceleration and velocity of 1 (and $i d e m$ for 2 ). Sometimes we pose $v_{12}^{i}=v_{1}^{i}-v_{2}^{i}$ for the relative velocity; and similarly for the relative position and acceleration. The equation for the other body is obtained by exchanging the labels $1 \leftrightarrow 2$ (remembering that $n_{12}^{i}$ changes sign in this operation).

As a consequence of the equivalence principle, which is incorporated "by hand" in Newton's theory and constitutes the fundamental basis of general relativity, the acceleration of 1 should 


\section{On the two-body problem in general relativity}

not depend on $m_{1}$ (nor on its internal structure), in the "test-mass" limit where the mass $m_{1}$ is much smaller than $m_{2}$. This is of course satisfied by the Newtonian acceleration (2), which is independent of $m_{1}$, but this leaves the possibility that the acceleration of the particle 1 , in higher approximations, does depend on $m_{1}$, via the so-called self-forces, which vanish in the test-mass limit. Indeed, this is what happens in the post-Newtonian corrections computed in Eqs. (3)-(6), which show explicitly many terms proportional to (powers of) $m_{1}$.

Though the successive post-Newtonian approximations are really a consequence of general relativity, they should be interpreted using the common-sense language of Newton. That is, having chosen a convenient general-relativistic (Cartesian) coordinate system, like the harmonic coordinate system adopted below, we should express the results in terms of the coordinate positions, velocities and accelerations of the bodies. Then, the trajectories of the particles can be viewed as taking place in the absolute Euclidean space of Newton, and their (coordinate) velocities as being defined with respect to absolute time. Not only this interpretation is the most satisfactory one from a conceptual point of view, but it represents also the most convenient path for comparing the theoretical predictions and the observations. For instance, the solar system dynamics at the first post-Newtonain level is defined, following a recent resolution of the International Astronomical Union, in a harmonic coordinate system, the Geocentric Reference System (GRS), with respect to which one considers the "absolute" motion of the planets and satellites.

With the same spirit we present below the 3PN equations of motion in terms of the harmoniccoordinate positions and velocities of the particles, i.e., in particular, in a form which is not manifestly covariant. But because the equations come from general relativity, they are endowed with the following properties, which make them truly "relativistic".

(I) The one-body problem in general relativity corresponds to the Schwarzschild solution, so the equations possess the correct "perturbative" limit, that given by the geodesics of the Schwarzschild metric (developed to the 3PN order), when the mass of one of the bodies tends to zero.

(II) Because general relativity admits the Poincaré group as a global symmetry (in the case of asymptotically flat space-times), the harmonic-coordinate equations of motion stay invariant when we perform a global Lorentz transformation, expanded at the 3PN order.

(III) Since the particles emit gravitational radiation there are some terms in the equations which are associated with radiation reaction. These terms appear at the order $2.5 \mathrm{PN}$ or $1 / c^{5}$ (where $5=2 s+1, s=2$ being the helicity of the graviton); see Eq. (11). They correspond to an "odd"order post-Newtonian correction, which does not stay invariant in a time reversal. By contrast, as we shall see, the "even"-orders 1PN, 2PN and 3PN correspond to a dynamics which is conservative.

(IV) General relativity is a non linear theory (even in vacuum), and some part of the gravitational radiation which was emitted by the particles in the past scatters off the static gravitational field generated by the rest-masses of the particles, or interacts gravitationally with itself. The "tail" radiation, produced by non-linear scattering, converges back onto the system at our present epoch, and modifies its current dynamics. The effect appears at the 4PN order [negligible in Eq. (1)], and corresponds to some "even"-order modification of the radiation-reaction damping [3].

The dominant relativistic correction term $\left(1 \mathrm{PN}\right.$, or $\left.1 / c^{2}\right)$ in the case of two non-spinning compact bodies was derived first by Lorentz and Droste 四. Subsequently, Einstein, Infeld and Hoffmann [5] obtained the 1PN corrections by means of their famous "surface-integral" method, in which the equations of motion are deduced from the vacuum field equations. This method is applicable to any compact objects (they be neutron stars, black holes, or, maybe, naked singularities). The $1 \mathrm{PN}$-accurate equations were also obtained, for the motion of the centers of mass of extended bodies, by Fock and followers [6], using a technique that can be qualified as more "physical" than 


\section{Luc Blanchet}

the surface-integral method, as it takes explicitly into account the internal structure of the bodies. The result is

$$
\begin{aligned}
A_{1 \mathrm{PN}}^{i} & =\left[5 \frac{G^{2} m_{1} m_{2}}{r_{12}^{3}}+4 \frac{G^{2} m_{2}^{2}}{r_{12}^{3}}+\frac{G m_{2}}{r_{12}^{2}}\left(\frac{3}{2}\left(n_{12} v_{2}\right)^{2}-v_{1}^{2}+4\left(v_{1} v_{2}\right)-2 v_{2}^{2}\right)\right] n_{12}^{i} \\
& +\frac{G m_{2}}{r_{12}^{2}}\left[4\left(n_{12} v_{1}\right)-3\left(n_{12} v_{2}\right)\right] v_{12}^{i} \cdot
\end{aligned}
$$

We denote the usual Euclidean scalar product by parenthesis, for instance $\left(n_{12} v_{1}\right)=\mathbf{n}_{12} \cdot \mathbf{v}_{1}$ and $\left(v_{1} v_{2}\right)=\mathbf{v}_{1} \cdot \mathbf{v}_{2}$. Witness the first term in Eq. (3), proportional to $m_{1}$, which represents a self-force at the $1 \mathrm{PN}$ order. The $1 \mathrm{PN}$-accurate equations of motion (known also for $N$ compact bodies) are sometimes called after the names of Einstein, Infeld and Hoffmann [5].

Things become more and more difficult when going to higher and higher post-Newtonian approximations. A technical problem is the rapid proliferation of terms. Typically any allowed term (compatible dimension, correct mass dependence) does appear with a definite non-zero coefficient in front. As a result, the expressions look quite complicated; that's maybe the price we have to pay for expressing in a Newtonian fashion what really is a relativistic equation. But, the point for our purpose is that the Newtonian-like equations are fully explicit: the successive relativistic corrections depend only on the instantaneous positions and velocities - all accelerations, appearing at intermediate stages of the calculation, are consistently "order-reduced" by means of the equations of motion themselves.

The 2PN approximation was tackled by Otha et al [7], how considered the post-Newtonian iteration of the Hamiltonian of $N$ point-particles. We refer here to the Hamiltonian as the Fokkertype Hamiltonian, which is obtained from the matter-plus-field Arnowitt-Deser-Misner (ADM)

Hamiltonian by eliminating the field degrees of freedom. The result for the 2PN equations of binary motion in harmonic coordinates was obtained by Damour and Deruelle [8, 9, 10], building on a non-linear iteration of the metric of two particles initiated in Ref. [11. It reads

$$
\begin{aligned}
A_{2 \mathrm{PN}}^{i} & =\left[-\frac{57}{4} \frac{G^{3} m_{1}^{2} m_{2}}{r_{12}^{4}}-\frac{69}{2} \frac{G^{3} m_{1} m_{2}^{2}}{r_{12}^{4}}-9 \frac{G^{3} m_{2}^{3}}{r_{12}^{4}}+\frac{G m_{2}}{r_{12}^{2}}\left(-\frac{15}{8}\left(n_{12} v_{2}\right)^{4}+\frac{3}{2}\left(n_{12} v_{2}\right)^{2} v_{1}^{2}\right.\right. \\
& \left.-6\left(n_{12} v_{2}\right)^{2}\left(v_{1} v_{2}\right)-2\left(v_{1} v_{2}\right)^{2}+\frac{9}{2}\left(n_{12} v_{2}\right)^{2} v_{2}^{2}+4\left(v_{1} v_{2}\right) v_{2}^{2}-2 v_{2}^{4}\right) \\
& +\frac{G^{2} m_{1} m_{2}}{r_{12}^{3}}\left(\frac{39}{2}\left(n_{12} v_{1}\right)^{2}-39\left(n_{12} v_{1}\right)\left(n_{12} v_{2}\right)+\frac{17}{2}\left(n_{12} v_{2}\right)^{2}-\frac{15}{4} v_{1}^{2}-\frac{5}{2}\left(v_{1} v_{2}\right)+\frac{5}{4} v_{2}^{2}\right) \\
& \left.+\frac{G^{2} m_{2}^{2}}{r_{12}^{3}}\left(2\left(n_{12} v_{1}\right)^{2}-4\left(n_{12} v_{1}\right)\left(n_{12} v_{2}\right)-6\left(n_{12} v_{2}\right)^{2}-8\left(v_{1} v_{2}\right)+4 v_{2}^{2}\right)\right] n_{12}^{i} \\
& +\left[\frac{G^{2} m_{2}^{2}}{r_{12}^{3}}\left(-2\left(n_{12} v_{1}\right)-2\left(n_{12} v_{2}\right)\right)+\frac{G^{2} m_{1} m_{2}}{r_{12}^{3}}\left(-\frac{63}{4}\left(n_{12} v_{1}\right)+\frac{55}{4}\left(n_{12} v_{2}\right)\right)\right. \\
& +\frac{G m_{2}}{r_{12}^{2}}\left(-6\left(n_{12} v_{1}\right)\left(n_{12} v_{2}\right)^{2}+\frac{9}{2}\left(n_{12} v_{2}\right)^{3}+\left(n_{12} v_{2}\right) v_{1}^{2}-4\left(n_{12} v_{1}\right)\left(v_{1} v_{2}\right)\right. \\
+ & \left.\left.4\left(n_{12} v_{2}\right)\left(v_{1} v_{2}\right)+4\left(n_{12} v_{1}\right) v_{2}^{2}-5\left(n_{12} v_{2}\right) v_{2}^{2}\right)\right] v_{12}^{i} .
\end{aligned}
$$

The corresponding result for the ADM-Hamiltonian of two particles at the 2PN order was given by Damour and Schäfer 12 . 


\section{On the two-body problem in general relativity}

As mentioned above, the equation of motion is conservative up to the $2 \mathrm{PN}$ level. Only at the $2.5 \mathrm{PN}$ order appears the first non-conservative effect, associated with the gravitational radiation reaction. The (harmonic-coordinate) equation of motion up to that level has been derived by Damour and Deruelle [8, 9, 10, who used it for the study of the radiation damping of the orbital period of the binary pulsar PSR $1913+16$ [10]. In addition, the 2.5PN-accurate ADM-Hamiltonian was worked out by Schäfer [13], and the harmonic-coordinate equations as well as the complete gravitational field of two particles were computed by Blanchet, Faye and Ponsot [14] by means of a new method coming from the work on gravitational-wave generation [1]. The latter calculations use a formal description of the objects by delta functions. Needless to say, there is mutual agreement between all the results obtained so far. The $2.5 \mathrm{PN}$ radiation damping term reads

$$
\begin{aligned}
A_{2.5 \mathrm{PN}}^{i} & =\frac{4}{5} \frac{G^{2} m_{1} m_{2}}{r_{12}^{3}}\left[-6 \frac{G m_{1}}{r_{12}}+\frac{52}{3} \frac{G m_{2}}{r_{12}}+3 v_{12}^{2}\right]\left(n_{12} v_{12}\right) n_{12}^{i} \\
& +\frac{4}{5} \frac{G^{2} m_{1} m_{2}}{r_{12}^{3}}\left[2 \frac{G m_{1}}{r_{12}}-8 \frac{G m_{2}}{r_{12}}-v_{12}^{2}\right] v_{12}^{i} .
\end{aligned}
$$

It is important to realize that the $2.5 \mathrm{PN}$ equations of motion (11)-(5) have been proved to hold in the case of binary systems of strongly self-gravitating bodies [10]. This is via an effacing principle of the internal structure of the bodies. As a result, the equations depend only on the "Schwarzschild" masses, $m_{1}$ and $m_{2}$, of the compact objects. Compacity parameters, namely the ratios $\frac{G m_{1}}{b_{1} c^{2}}$ and $\frac{G m_{2}}{b_{2} c^{2}}$ between the masses and the radii $b_{1}$ and $b_{2}$ of the objects, do not enter the equations of motion. This fact has been explicitly verified at the 2.5PN order by Grishchuk and Kopejkin [15], who have made a "physical" computation, à la Fock, taking into account the internal structure of two self-gravitating extended bodies. Furthermore, the same 2.5PN equations of motion have also been established by Itoh, Futamase and Asada [16], who use a variant of the surface-integral approach [5], valid for compact bodies, independently of the strength of the internal gravity.

The present state of the art is the $3 \mathrm{PN}$ approximation. The equations to this order have been worked out independently by two groups, by means of different methods, and with equivalent results. On one hand, Jaranowski and Schäfer [17], and Damour, Jaranowski and Schäfer [18], following the line of research of Refs. [7, 12, 13], employ the ADM-Hamiltonian formalism of general relativity; on the other hand, Blanchet and Faye [20, 21], and Andrade, Blanchet and Faye [22], founding their approach on the post-Newtonian iteration initiated in Ref. [14], compute directly the harmonic-coordinate equations of motion. The end results are physically equivalent in the sense that there exists a unique "contact" transformation of the dynamical variables, that changes the harmonic-coordinate Lagrangian obtained in Ref. 222 into a new Lagrangian, whose associated Hamiltonian coincides exactly with the one given in Ref. [18]. The 3PN contribution to the acceleration of the particle 1 reads, in a Cartesian harmonic coordinate system,

$$
\begin{aligned}
A_{3 \mathrm{PN}}^{i} & =\left[\frac { G m _ { 2 } } { r _ { 1 2 } ^ { 2 } } \left(\frac{35}{16}\left(n_{12} v_{2}\right)^{6}-\frac{15}{8}\left(n_{12} v_{2}\right)^{4} v_{1}^{2}+\frac{15}{2}\left(n_{12} v_{2}\right)^{4}\left(v_{1} v_{2}\right)+3\left(n_{12} v_{2}\right)^{2}\left(v_{1} v_{2}\right)^{2}\right.\right. \\
& -\frac{15}{2}\left(n_{12} v_{2}\right)^{4} v_{2}^{2}+\frac{3}{2}\left(n_{12} v_{2}\right)^{2} v_{1}^{2} v_{2}^{2}-12\left(n_{12} v_{2}\right)^{2}\left(v_{1} v_{2}\right) v_{2}^{2}-2\left(v_{1} v_{2}\right)^{2} v_{2}^{2} \\
& \left.+\frac{15}{2}\left(n_{12} v_{2}\right)^{2} v_{2}^{4}+4\left(v_{1} v_{2}\right) v_{2}^{4}-2 v_{2}^{6}\right)+\frac{G^{2} m_{1} m_{2}}{r_{12}^{3}}\left(-\frac{171}{8}\left(n_{12} v_{1}\right)^{4}\right. \\
& +\frac{171}{2}\left(n_{12} v_{1}\right)^{3}\left(n_{12} v_{2}\right)-\frac{723}{4}\left(n_{12} v_{1}\right)^{2}\left(n_{12} v_{2}\right)^{2}+\frac{383}{2}\left(n_{12} v_{1}\right)\left(n_{12} v_{2}\right)^{3} \\
& -\frac{455}{8}\left(n_{12} v_{2}\right)^{4}+\frac{229}{4}\left(n_{12} v_{1}\right)^{2} v_{1}^{2}-\frac{205}{2}\left(n_{12} v_{1}\right)\left(n_{12} v_{2}\right) v_{1}^{2}+\frac{191}{4}\left(n_{12} v_{2}\right)^{2} v_{1}^{2}-\frac{91}{8} v_{1}^{4}
\end{aligned}
$$




\section{Luc Blanchet}

$$
\begin{aligned}
& -\frac{229}{2}\left(n_{12} v_{1}\right)^{2}\left(v_{1} v_{2}\right)+244\left(n_{12} v_{1}\right)\left(n_{12} v_{2}\right)\left(v_{1} v_{2}\right)-\frac{225}{2}\left(n_{12} v_{2}\right)^{2}\left(v_{1} v_{2}\right) \\
& +\frac{91}{2} v_{1}^{2}\left(v_{1} v_{2}\right)-\frac{177}{4}\left(v_{1} v_{2}\right)^{2}+\frac{229}{4}\left(n_{12} v_{1}\right)^{2} v_{2}^{2}-\frac{283}{2}\left(n_{12} v_{1}\right)\left(n_{12} v_{2}\right) v_{2}^{2} \\
& \left.+\frac{259}{4}\left(n_{12} v_{2}\right)^{2} v_{2}^{2}-\frac{91}{4} v_{1}^{2} v_{2}^{2}+43\left(v_{1} v_{2}\right) v_{2}^{2}-\frac{81}{8} v_{2}^{4}\right)+\frac{G^{2} m_{2}^{2}}{r_{12}^{3}}\left(-6\left(n_{12} v_{1}\right)^{2}\left(n_{12} v_{2}\right)^{2}\right. \\
& +12\left(n_{12} v_{1}\right)\left(n_{12} v_{2}\right)^{3}+6\left(n_{12} v_{2}\right)^{4}+4\left(n_{12} v_{1}\right)\left(n_{12} v_{2}\right)\left(v_{1} v_{2}\right)+12\left(n_{12} v_{2}\right)^{2}\left(v_{1} v_{2}\right) \\
& \left.+4\left(v_{1} v_{2}\right)^{2}-4\left(n_{12} v_{1}\right)\left(n_{12} v_{2}\right) v_{2}^{2}-12\left(n_{12} v_{2}\right)^{2} v_{2}^{2}-8\left(v_{1} v_{2}\right) v_{2}^{2}+4 v_{2}^{4}\right) \\
& +\frac{G^{3} m_{2}^{3}}{r_{12}^{4}}\left(-\left(n_{12} v_{1}\right)^{2}+2\left(n_{12} v_{1}\right)\left(n_{12} v_{2}\right)+\frac{43}{2}\left(n_{12} v_{2}\right)^{2}+18\left(v_{1} v_{2}\right)-9 v_{2}^{2}\right) \\
& +\frac{G^{3} m_{1} m_{2}^{2}}{r_{12}^{4}}\left(\frac{415}{8}\left(n_{12} v_{1}\right)^{2}-\frac{375}{4}\left(n_{12} v_{1}\right)\left(n_{12} v_{2}\right)+\frac{1113}{8}\left(n_{12} v_{2}\right)^{2}\right. \\
& \left.-\frac{615}{64}\left(n_{12} v_{12}\right)^{2} \pi^{2}+\frac{123}{64} v_{12}^{2} \pi^{2}+18 v_{1}^{2}+33\left(v_{1} v_{2}\right)-\frac{33}{2} v_{2}^{2}\right) \\
& +\frac{G^{3} m_{1}^{2} m_{2}}{r_{12}^{4}}\left(-\frac{45887}{168}\left(n_{12} v_{1}\right)^{2}+\frac{24025}{42}\left(n_{12} v_{1}\right)\left(n_{12} v_{2}\right)-\frac{10469}{42}\left(n_{12} v_{2}\right)^{2}\right. \\
& \left.+\frac{48197}{840} v_{1}^{2}-\frac{36227}{420}\left(v_{1} v_{2}\right)+\frac{36227}{840} v_{2}^{2}+110\left(n_{12} v_{12}\right)^{2} \ln \left(\frac{r_{12}}{r_{1}^{\prime}}\right)-22 v_{12}^{2} \ln \left(\frac{r_{12}}{r_{1}^{\prime}}\right)\right) \\
& +\frac{G^{4} m_{1}^{3} m_{2}}{r_{12}^{5}}\left(-\frac{3187}{1260}+\frac{44}{3} \ln \left(\frac{r_{12}}{r_{1}^{\prime}}\right)\right)+\frac{G^{4} m_{1}^{2} m_{2}^{2}}{r_{12}^{5}}\left(\frac{34763}{210}-\frac{44}{3} \lambda-\frac{41}{16} \pi^{2}\right) \\
& \left.+\frac{G^{4} m_{1} m_{2}^{3}}{r_{12}^{5}}\left(\frac{10478}{63}-\frac{44}{3} \lambda-\frac{41}{16} \pi^{2}-\frac{44}{3} \ln \left(\frac{r_{12}}{r_{2}^{\prime}}\right)\right)+16 \frac{G^{4} m_{2}^{4}}{r_{12}^{5}}\right] n_{12}^{i} \\
& +\left[\frac { G m _ { 2 } } { r _ { 1 2 } ^ { 2 } } \left(\frac{15}{2}\left(n_{12} v_{1}\right)\left(n_{12} v_{2}\right)^{4}-\frac{45}{8}\left(n_{12} v_{2}\right)^{5}-\frac{3}{2}\left(n_{12} v_{2}\right)^{3} v_{1}^{2}+6\left(n_{12} v_{1}\right)\left(n_{12} v_{2}\right)^{2}\left(v_{1} v_{2}\right)\right.\right. \\
& -6\left(n_{12} v_{2}\right)^{3}\left(v_{1} v_{2}\right)-2\left(n_{12} v_{2}\right)\left(v_{1} v_{2}\right)^{2}-12\left(n_{12} v_{1}\right)\left(n_{12} v_{2}\right)^{2} v_{2}^{2}+12\left(n_{12} v_{2}\right)^{3} v_{2}^{2} \\
& \left.+\left(n_{12} v_{2}\right) v_{1}^{2} v_{2}^{2}-4\left(n_{12} v_{1}\right)\left(v_{1} v_{2}\right) v_{2}^{2}+8\left(n_{12} v_{2}\right)\left(v_{1} v_{2}\right) v_{2}^{2}+4\left(n_{12} v_{1}\right) v_{2}^{4}-7\left(n_{12} v_{2}\right) v_{2}^{4}\right) \\
& +\frac{G^{2} m_{2}^{2}}{r_{12}^{3}}\left(-2\left(n_{12} v_{1}\right)^{2}\left(n_{12} v_{2}\right)+8\left(n_{12} v_{1}\right)\left(n_{12} v_{2}\right)^{2}+2\left(n_{12} v_{2}\right)^{3}\right. \\
& \left.+2\left(n_{12} v_{1}\right)\left(v_{1} v_{2}\right)+4\left(n_{12} v_{2}\right)\left(v_{1} v_{2}\right)-2\left(n_{12} v_{1}\right) v_{2}^{2}-4\left(n_{12} v_{2}\right) v_{2}^{2}\right) \\
& +\frac{G^{2} m_{1} m_{2}}{r_{12}^{3}}\left(-\frac{243}{4}\left(n_{12} v_{1}\right)^{3}+\frac{565}{4}\left(n_{12} v_{1}\right)^{2}\left(n_{12} v_{2}\right)-\frac{269}{4}\left(n_{12} v_{1}\right)\left(n_{12} v_{2}\right)^{2}\right. \\
& -\frac{95}{12}\left(n_{12} v_{2}\right)^{3}+\frac{207}{8}\left(n_{12} v_{1}\right) v_{1}^{2}-\frac{137}{8}\left(n_{12} v_{2}\right) v_{1}^{2}-36\left(n_{12} v_{1}\right)\left(v_{1} v_{2}\right) \\
& \left.+\frac{27}{4}\left(n_{12} v_{2}\right)\left(v_{1} v_{2}\right)+\frac{81}{8}\left(n_{12} v_{1}\right) v_{2}^{2}+\frac{83}{8}\left(n_{12} v_{2}\right) v_{2}^{2}\right)+\frac{G^{3} m_{2}^{3}}{r_{12}^{4}}\left(4\left(n_{12} v_{1}\right)+5\left(n_{12} v_{2}\right)\right) \\
& +\frac{G^{3} m_{1} m_{2}^{2}}{r_{12}^{4}}\left(-\frac{307}{8}\left(n_{12} v_{1}\right)+\frac{479}{8}\left(n_{12} v_{2}\right)+\frac{123}{32}\left(n_{12} v_{12}\right) \pi^{2}\right) \\
& \left.+\frac{G^{3} m_{1}^{2} m_{2}}{r_{12}^{4}}\left(\frac{31397}{420}\left(n_{12} v_{1}\right)-\frac{36227}{420}\left(n_{12} v_{2}\right)-44\left(n_{12} v_{12}\right) \ln \left(\frac{r_{12}}{r_{1}^{\prime}}\right)\right)\right] v_{12}^{i} \text {. }
\end{aligned}
$$




\section{On the two-body problem in general relativity}

Notice that the 3PN term involves some $\pi^{2}$. Typically, the $\pi^{2}$ terms arise from non-linear interactions involving some integrals such as $\frac{1}{\pi} \int \frac{d^{3} \mathrm{x}}{r_{1}^{2} r_{2}^{2}}=\frac{\pi^{2}}{r_{12}}$.

More importantly, we see that the $3 \mathrm{PN}$ acceleration depends on three arbitrary constants: two length scales $r_{1}^{\prime}$ and $r_{2}^{\prime}$ entering some logarithms, and a dimensionless constant $\lambda$. It was proved in Ref. 20] that $r_{1}^{\prime}$ and $r_{2}^{\prime}$ are merely linked with the choice of harmonic coordinates. Indeed, as we are using point-particles, the usual condition of harmonic coordinates, viz $\partial_{\nu} h^{\mu \nu}=0$, does not completely fix the gauge, as we can always add a gauge vector $\xi^{\mu}=\delta x^{\mu}$, which satisfies $\Delta \xi^{\mu}=0$ and is singular at the location of the two particles. The constants $r_{1}^{\prime}$ and $r_{2}^{\prime}$ can thus be removed by a coordinate transformation; as a result they will never appear in any physical result, such as the invariant center-of-mass energy of the binary.

By contrast with the harmless gauge-constants $r_{1}^{\prime}$ and $r_{2}^{\prime}$, the constant $\lambda$ represents a true physical ambiguity, which reflects probably an incompleteness of the Hadamard regularization used to cope with the infinite self-field of point-particles. This regularization is based on Hadamard's concept of the "partie finie" of singular functions and divergent integrals. Actually, it has been found necessary for solving this problem to develop an extended version of the Hadamard regularization, and a theory of generalized functions associated with it [21]. Very likely, the presence of $\lambda$ is related to the fact that, starting from the $3 \mathrm{PN}$ order, many non-linear integrals composing the equations of motion, when taken separately, depend on the internal structure of each body, even in the limit where the radius tends to zero. The results given by the ADM-Hamiltonian approach [17, 18] depend also on one arbitrary physical parameter, called $\omega_{\text {static }}$. More precisely, the authors [17] introduced originally two unknown constants, $\omega_{\text {static }}$ and $\omega_{\text {kinetic }}$, but $\omega_{\text {kinetic }}$ was fixed later to a unique value by imposing, in an ad hoc manner, the global Poincaré invariance of the Hamiltonian [18]. On the other hand, the authors [20, 21] have only one constant $\lambda$ because their regularization is defined in a Lorentz-invariant way. The equivalence between the approaches [20, 21, 22] and 17, 18 holds if and only if $\lambda=-\frac{3}{11} \omega_{\text {static }}-\frac{1987}{3080}$. Recently, the value $\omega_{\text {static }}=0$ has been obtained by means of a different regularization (dimensional) within the ADM-Hamiltonian approach [19]. This result would mean that $\lambda=-\frac{1987}{3080}$ (but we keep $\lambda$ unspecified in the present discussion).

Going to still higher post-Newtonian orders, we mention that the subdominant radiation-reaction effect at the 3.5PN order has been calculated in Ref. [23] for two bodies in an arbitrary gauge, and in Ref. [24 for general "fluids" in a Burke-Thorne-extended gauge (there is agreement between the two methods [23]). Also, we know the contribution of the tails of waves in the equations of motion, which appears at the $4 \mathrm{PN}$, or $1 / c^{8}$, order [3].

The complicated expressions (1)-(6) simplify drastically in the case of an orbit which is circular, apart from the gradual inspiral driven by radiation reaction. This case corresponds to the physical situation of most inspiralling compact binaries, since the radiation reaction forces tend to circularize rapidly the orbit. In this case the relative acceleration reads

$$
a_{12}^{i}=-\omega^{2} y_{12}^{i}-\frac{32}{5} \frac{G^{3} m^{3} \nu}{c^{5} r_{12}^{4}} v_{12}^{i}+\mathcal{O}\left(\frac{1}{c^{7}}\right)
$$

Relative quantities are denoted e.g. by $y_{12}^{i}=y_{1}^{i}-y_{2}^{i}$; mass parameters are the total mass $m=$ $m_{1}+m_{2}$ and the mass ratio $\nu=m_{1} m_{2} / m^{2}$ such that $0<\nu \leq \frac{1}{4}$, with $\nu=\frac{1}{4}$ when the two masses are equal, and $\nu \rightarrow 0$ in the test-mass limit for one of the particles. The second term in Eq. (7) is the radiation reaction force, whose expression follows immediately from Eq. (5), while $\omega$ represents the orbital frequency of the circular motion at the $3 \mathrm{PN}$ order, and is related to the harmonic-coordinate separation $r_{12}$ by the "Kepler" law 


\section{Luc Blanchet}

$$
\begin{aligned}
\omega^{2} & =\frac{G m}{r_{12}^{3}}\left\{1+(-3+\nu) \gamma+\left(6+\frac{41}{4} \nu+\nu^{2}\right) \gamma^{2}\right. \\
& \left.+\left(-10+\left[-\frac{67759}{840}+\frac{41}{64} \pi^{2}+22 \ln \left(\frac{r}{r_{0}^{\prime}}\right)+\frac{44}{3} \lambda\right] \nu+\frac{19}{2} \nu^{2}+\nu^{3}\right) \gamma^{3}\right\} .
\end{aligned}
$$

The post-Newtonian corrections are parametrized by $\gamma=\frac{G m}{r_{12} c^{2}}$. The constant $r_{0}^{\prime}$ is given by $\ln r_{0}^{\prime}=$ $\frac{m_{1}}{m} \ln r_{1}^{\prime}+\frac{m_{2}}{m} \ln r_{2}^{\prime}$, where $r_{1}^{\prime}$ and $r_{2}^{\prime}$ are the two gauge-constants in Eq. (6). The circular-orbit equation (7)-(8) is used, notably, to compute the third time-derivative of the binary's quadrupole moment, which constitutes the main contribution to the gravitational-wave flux at infinity, at the $3 \mathrm{PN}$ order [1].

Finally, coming back to the general case of non-circular orbits, we show that the $3 \mathrm{PN}$ equations of motion, when neglecting the radiation-reaction term at the 2.5PN order, admit a Euler-Lagrange formulation. As it turns out, the Lagrangian in harmonic coordinates is a generalized one, in the sense that it depends not only on the positions and velocities of the particles, like an ordinary Lagrangian, but also, starting at the $2 \mathrm{PN}$ order, on their accelerations. This fact has been established in Refs. [8, 10, 12]. Thus, the equations of motion take the form

$$
\frac{\partial L}{\partial y_{1}^{i}}-\frac{d}{d t}\left(\frac{\partial L}{\partial v_{1}^{i}}\right)+\frac{d^{2}}{d t^{2}}\left(\frac{\partial L}{\partial a_{1}^{i}}\right)+\frac{1}{c^{5}} m_{1} A_{2.5 \mathrm{PN}}^{i}=\mathcal{O}\left(\frac{1}{c^{7}}\right)
$$

where $A_{2.5 \mathrm{PN}}^{i}$ is given by Eq. (5), and where the 3PN harmonic-coordinate generalized Lagrangian is of the type

$$
L=L_{\mathrm{N}}+\frac{1}{c^{2}} L_{1 \mathrm{PN}}+\frac{1}{c^{4}} L_{2 \mathrm{PN}}+\frac{1}{c^{6}} L_{3 \mathrm{PN}}+\mathcal{O}\left(\frac{1}{c^{8}}\right) .
$$

The Newtonian piece reads

$$
L_{\mathrm{N}}=\frac{m_{1} v_{1}^{2}}{2}+\frac{m_{2} v_{2}^{2}}{2}+\frac{G m_{1} m_{2}}{r_{12}} .
$$

The 1PN piece, which is still "ordinary" (not depending on accelerations), has been obtained by Lorentz and Droste [4], and Fichtenholz [25]:

$$
\begin{aligned}
L_{1 \mathrm{PN}} & =\frac{m_{1} v_{1}^{4}}{8}+\frac{G m_{1} m_{2}}{r_{12}}\left(-\frac{1}{4}\left(n_{12} v_{1}\right)\left(n_{12} v_{2}\right)+\frac{3}{2} v_{1}^{2}-\frac{7}{4}\left(v_{1} v_{2}\right)\right) \\
& -\frac{G^{2} m_{1}^{2} m_{2}}{2 r_{12}^{2}}+1 \leftrightarrow 2 .
\end{aligned}
$$

To the expression given above, one must add the terms corresponding to the label exchange $1 \leftrightarrow 2$, including those that are already symmetric under exchange. Now, starting at the $2 \mathrm{PN}$ order, we get a dependence over the accelerations [8, 10, 12]. By adding to the Lagrangian some so-called "multi-zero" terms, which do not contribute to the equations of motion, one can always arrange that the dependence over the accelerations be linear. Of course, it is not allowed to replace the accelerations by the equations of motion in a Lagrangian (however, this can and should be done in the final expressions of the conserved integrals derived from that Lagrangian). We get 


$$
\begin{aligned}
L_{2 \mathrm{PN}} & =\frac{m_{1} v_{1}^{6}}{16}+\frac{1}{2} \frac{G^{3} m_{1}^{3} m_{2}}{r_{12}^{3}}+\frac{19}{8} \frac{G^{3} m_{1}^{2} m_{2}^{2}}{r_{12}^{3}}+\frac{G^{2} m_{1}^{2} m_{2}}{r_{12}^{2}}\left(\frac{7}{2}\left(n_{12} v_{1}\right)^{2}-\frac{7}{2}\left(n_{12} v_{1}\right)\left(n_{12} v_{2}\right)\right. \\
& \left.+\frac{1}{2}\left(n_{12} v_{2}\right)^{2}+\frac{1}{4} v_{1}^{2}-\frac{7}{4}\left(v_{1} v_{2}\right)+\frac{7}{4} v_{2}^{2}\right)+\frac{G m_{1} m_{2}}{r_{12}}\left(\frac{3}{16}\left(n_{12} v_{1}\right)^{2}\left(n_{12} v_{2}\right)^{2}\right. \\
& \left.-\frac{7}{8}\left(n_{12} v_{2}\right)^{2} v_{1}^{2}+\frac{7}{8} v_{1}^{4}+\frac{3}{4}\left(n_{12} v_{1}\right)\left(n_{12} v_{2}\right)\left(v_{1} v_{2}\right)-2 v_{1}^{2}\left(v_{1} v_{2}\right)+\frac{1}{8}\left(v_{1} v_{2}\right)^{2}+\frac{15}{16} v_{1}^{2} v_{2}^{2}\right) \\
& +G m_{1} m_{2}\left(-\frac{7}{4}\left(a_{1} v_{2}\right)\left(n_{12} v_{2}\right)-\frac{1}{8}\left(n_{12} a_{1}\right)\left(n_{12} v_{2}\right)^{2}+\frac{7}{8}\left(n_{12} a_{1}\right) v_{2}^{2}\right)+1 \leftrightarrow 2 .
\end{aligned}
$$

Finally, the 3PN-accurate piece of the harmonic-coordinate Lagrangian depends also on accelerations; it is notable that accelerations are sufficient, there is no need to include, at the 3PN order, derivatives of accelerations. We find 22]

$$
\begin{aligned}
& L_{3 \mathrm{PN}}=\frac{5}{128} m_{1} v_{1}^{8}+\frac{G^{2} m_{1}^{2} m_{2}}{r_{12}^{2}}\left(\frac{13}{18}\left(n_{12} v_{1}\right)^{4}+\frac{83}{18}\left(n_{12} v_{1}\right)^{3}\left(n_{12} v_{2}\right)-\frac{35}{6}\left(n_{12} v_{1}\right)^{2}\left(n_{12} v_{2}\right)^{2}\right. \\
& -\frac{245}{24}\left(n_{12} v_{1}\right)^{2} v_{1}^{2}+\frac{179}{12}\left(n_{12} v_{1}\right)\left(n_{12} v_{2}\right) v_{1}^{2}-\frac{235}{24}\left(n_{12} v_{2}\right)^{2} v_{1}^{2}+\frac{373}{48} v_{1}^{4}+\frac{529}{24}\left(n_{12} v_{1}\right)^{2}\left(v_{1} v_{2}\right) \\
& -\frac{97}{6}\left(n_{12} v_{1}\right)\left(n_{12} v_{2}\right)\left(v_{1} v_{2}\right)-\frac{719}{24} v_{1}^{2}\left(v_{1} v_{2}\right)+\frac{463}{24}\left(v_{1} v_{2}\right)^{2}-\frac{7}{24}\left(n_{12} v_{1}\right)^{2} v_{2}^{2} \\
& \left.-\frac{1}{2}\left(n_{12} v_{1}\right)\left(n_{12} v_{2}\right) v_{2}^{2}+\frac{1}{4}\left(n_{12} v_{2}\right)^{2} v_{2}^{2}+\frac{463}{48} v_{1}^{2} v_{2}^{2}-\frac{19}{2}\left(v_{1} v_{2}\right) v_{2}^{2}+\frac{45}{16} v_{2}^{4}\right) \\
& +G m_{1} m_{2}\left(\frac{3}{8}\left(a_{1} v_{2}\right)\left(n_{12} v_{1}\right)\left(n_{12} v_{2}\right)^{2}+\frac{5}{12}\left(a_{1} v_{2}\right)\left(n_{12} v_{2}\right)^{3}+\frac{1}{8}\left(n_{12} a_{1}\right)\left(n_{12} v_{1}\right)\left(n_{12} v_{2}\right)^{3}\right. \\
& +\frac{1}{16}\left(n_{12} a_{1}\right)\left(n_{12} v_{2}\right)^{4}+\frac{11}{4}\left(a_{1} v_{1}\right)\left(n_{12} v_{2}\right) v_{1}^{2}-\left(a_{1} v_{2}\right)\left(n_{12} v_{2}\right) v_{1}^{2}-2\left(a_{1} v_{1}\right)\left(n_{12} v_{2}\right)\left(v_{1} v_{2}\right) \\
& +\frac{1}{4}\left(a_{1} v_{2}\right)\left(n_{12} v_{2}\right)\left(v_{1} v_{2}\right)+\frac{3}{8}\left(n_{12} a_{1}\right)\left(n_{12} v_{2}\right)^{2}\left(v_{1} v_{2}\right)-\frac{5}{8}\left(n_{12} a_{1}\right)\left(n_{12} v_{1}\right)^{2} v_{2}^{2} \\
& +\frac{15}{8}\left(a_{1} v_{1}\right)\left(n_{12} v_{2}\right) v_{2}^{2}-\frac{15}{8}\left(a_{1} v_{2}\right)\left(n_{12} v_{2}\right) v_{2}^{2}-\frac{1}{2}\left(n_{12} a_{1}\right)\left(n_{12} v_{1}\right)\left(n_{12} v_{2}\right) v_{2}^{2} \\
& \left.-\frac{5}{16}\left(n_{12} a_{1}\right)\left(n_{12} v_{2}\right)^{2} v_{2}^{2}\right)+\frac{G^{2} m_{1}^{2} m_{2}}{r_{12}}\left(-\frac{235}{24}\left(a_{2} v_{1}\right)\left(n_{12} v_{1}\right)-\frac{29}{24}\left(n_{12} a_{2}\right)\left(n_{12} v_{1}\right)^{2}\right. \\
& -\frac{235}{24}\left(a_{1} v_{2}\right)\left(n_{12} v_{2}\right)-\frac{17}{6}\left(n_{12} a_{1}\right)\left(n_{12} v_{2}\right)^{2}+\frac{185}{16}\left(n_{12} a_{1}\right) v_{1}^{2}-\frac{235}{48}\left(n_{12} a_{2}\right) v_{1}^{2} \\
& \left.-\frac{185}{8}\left(n_{12} a_{1}\right)\left(v_{1} v_{2}\right)+\frac{20}{3}\left(n_{12} a_{1}\right) v_{2}^{2}\right)+\frac{G m_{1} m_{2}}{r_{12}}\left(-\frac{5}{32}\left(n_{12} v_{1}\right)^{3}\left(n_{12} v_{2}\right)^{3}\right. \\
& +\frac{1}{8}\left(n_{12} v_{1}\right)\left(n_{12} v_{2}\right)^{3} v_{1}^{2}+\frac{5}{8}\left(n_{12} v_{2}\right)^{4} v_{1}^{2}-\frac{11}{16}\left(n_{12} v_{1}\right)\left(n_{12} v_{2}\right) v_{1}^{4}+\frac{1}{4}\left(n_{12} v_{2}\right)^{2} v_{1}^{4}+\frac{11}{16} v_{1}^{6} \\
& -\frac{15}{32}\left(n_{12} v_{1}\right)^{2}\left(n_{12} v_{2}\right)^{2}\left(v_{1} v_{2}\right)+\left(n_{12} v_{1}\right)\left(n_{12} v_{2}\right) v_{1}^{2}\left(v_{1} v_{2}\right)+\frac{3}{8}\left(n_{12} v_{2}\right)^{2} v_{1}^{2}\left(v_{1} v_{2}\right) \\
& -\frac{13}{16} v_{1}^{4}\left(v_{1} v_{2}\right)+\frac{5}{16}\left(n_{12} v_{1}\right)\left(n_{12} v_{2}\right)\left(v_{1} v_{2}\right)^{2}+\frac{1}{16}\left(v_{1} v_{2}\right)^{3}-\frac{5}{8}\left(n_{12} v_{1}\right)^{2} v_{1}^{2} v_{2}^{2} \\
& \text { - } \left.\frac{23}{32}\left(n_{12} v_{1}\right)\left(n_{12} v_{2}\right) v_{1}^{2} v_{2}^{2}+\frac{1}{16} v_{1}^{4} v_{2}^{2}-\frac{1}{32} v_{1}^{2}\left(v_{1} v_{2}\right) v_{2}^{2}\right) \\
& -\frac{3}{8} \frac{G^{4} m_{1}^{4} m_{2}}{r_{12}^{4}}+\frac{G^{4} m_{1}^{3} m_{2}^{2}}{r_{12}^{4}}\left(-\frac{5809}{280}+\frac{11}{3} \lambda+\frac{22}{3} \ln \left(\frac{r_{12}}{r_{1}^{\prime}}\right)\right)
\end{aligned}
$$




\section{Luc Blanchet}

$$
\begin{aligned}
& +\frac{G^{3} m_{1}^{2} m_{2}^{2}}{r_{12}^{3}}\left(\frac{383}{24}\left(n_{12} v_{1}\right)^{2}-\frac{889}{48}\left(n_{12} v_{1}\right)\left(n_{12} v_{2}\right)-\frac{305}{72} v_{1}^{2}+\frac{439}{144}\left(v_{1} v_{2}\right)\right. \\
& \left.-\frac{123}{64}\left(n_{12} v_{1}\right)^{2} \pi^{2}+\frac{123}{64}\left(n_{12} v_{1}\right)\left(n_{12} v_{2}\right) \pi^{2}+\frac{41}{64} v_{1}^{2} \pi^{2}-\frac{41}{64}\left(v_{1} v_{2}\right) \pi^{2}\right) \\
& +\frac{G^{3} m_{1}^{3} m_{2}}{r_{12}^{3}}\left(-\frac{8243}{210}\left(n_{12} v_{1}\right)^{2}+\frac{15541}{420}\left(n_{12} v_{1}\right)\left(n_{12} v_{2}\right)+\frac{3}{2}\left(n_{12} v_{2}\right)^{2}\right. \\
& +\frac{15611}{1260} v_{1}^{2}-\frac{17501}{1260}\left(v_{1} v_{2}\right)+\frac{5}{4} v_{2}^{2}+22\left(n_{12} v_{1}\right)\left(n_{12} v_{12}\right) \ln \left(\frac{r_{12}}{r_{1}^{\prime}}\right) \\
& \left.-\frac{22}{3}\left(v_{1} v_{12}\right) \ln \left(\frac{r_{12}}{r_{1}^{\prime}}\right)\right)+1 \leftrightarrow 2 .
\end{aligned}
$$

See Ref. 22 for the explicit expressions of the ten conserved quantities, at the 3PN order, corresponding to the integrals of energy, linear and angular momentum, and center-of-mass position.

\section{References}

[1] L. Blanchet, B.R. Iyer and B. Joguet, Phys. Rev. D, submitted (gr-qc/0105098).

[2] L. Blanchet, G. Faye, B.R. Iyer and B. Joguet, Phys. Rev. Lett., submitted (gr-qc/0105099).

[3] L. Blanchet and T. Damour, Phys. Rev. D37, 1410 (1988).

[4] H.A. Lorentz and J.Droste, Versl. K. Akad. Wet. Amsterdam 26, 392 and 649 (1917); in the collected papers of H.A. Lorentz, vol. 5, The Hague, Nijhoff (1937).

[5] A. Einstein, L. Infeld and B. Hoffmann, Ann. Math. 39, 65 (1938); A. Einstein and L. Infeld, Ann. Math. 41, 797 (1940); Can. J. Math. 1, 209 (1949).

[6] V. Fock, J. Phys. (U.S.S.R.) 1, 81 (1939); N. Petrova, J. Phys. (U.S.S.R.) 19, 989 (1949); A. Papapetrou, Proc. Phys. Soc. (London) 64, 57 (1951).

[7] T. Ohta, H. Okamura, T. Kimura and K. Hiida, Progr. Theor. Phys. 50, 492 (1973); Progr. Theor. Phys. 51, 1220 (1974);Progr. Theor. Phys. 51, 1598 (1974).

[8] T. Damour and N. Deruelle, Phys. Lett. 87A, 81 (1981); C. R. Acad. Sc. Paris, 293, 537 (1981).

[9] T. Damour, C. R. Acad. Sc. Paris, 294, 1355 (1982).

[10] T. Damour, in Gravitational Radiation, N. Deruelle and T. Piran (eds.), North-Holland Company (1983); Phys. Rev. Lett. 51, 1019 (1983).

[11] L. Bel, T. Damour, N. Deruelle, J. Ibañez and J. Martin, Gen. Relativ. Gravit. 13, 963 (1981).

[12] T. Damour and G. Schäfer, Gen. Rel. Grav. 17, 879 (1985).

[13] G. Schäfer, Ann. Phys. (N.Y.) 161, 81 (1985); Gen. Rel. Grav. 18, 255 (1986).

[14] L. Blanchet, G. Faye and B. Ponsot, Phys. Rev. D58, 124002 (1998).

[15] S.M. Kopejkin, Astron. Zh. 62, 889 (1985); L.P. Grishchuk and S.M. Kopejkin, in Relativity in Celestial Mechanics and Astrometry, J. Kovalevsky and V.A. Brumberg (eds.), Reidel, Dordrecht (1986).

[16] Y. Itoh, T. Futamase and H. Asada, Phys. Rev. D62, 064002 (2000); Phys. Rev. D63, 064038 (2001).

[17] P. Jaranowski and G. Schäfer, Phys. Rev. D57, 7274 (1998); Phys. Rev. D60, 124003 (1999); Annalen Phys. 9, 378 (2000).

[18] T. Damour, P. Jaranowski and G. Schäfer, Phys. Rev. D62, 021501R (2000), Erratum Phys. Rev. D63, 029903 (2001); Phys. Rev. D63, 044021 (2001).

[19] T. Damour, P. Jaranowski and G. Schäfer, gr-qc/015038.

[20] L. Blanchet and G. Faye, Phys. Lett. A 271, 58 (2000); Phys. Rev. D63, 062005 (2001).

[21] L. Blanchet and G. Faye, J. Math. Phys. 41, 7675 (2000); J. Math. Phys., in press (gr-qc/0006100).

[22] V. C. de Andrade, L. Blanchet and G. Faye, Class. Quantum Grav. 18, 753 (2001).

[23] B.R. Iyer and C.M. Will, Phys. Rev. Lett. 70, 113 (1993); Phys. Rev. D52, 6882 (1995).

[24] L. Blanchet, Phys. Rev. D47, 4392 (1993); Phys. Rev. D55, 714 (1997).

[25] I.G. Fichtenholz, Zh. Eksp. Teor. Fiz. 20, 233 (1950). 